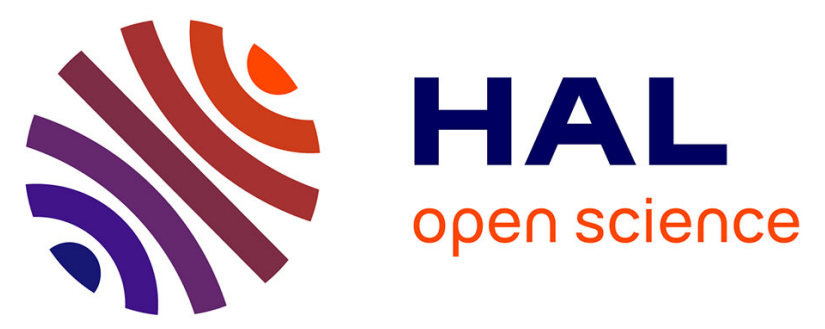

\title{
Peut-on mimer la digestion gastrique du nouveau-né grâce à un modèle in vitro dynamique ?
}

Samira Cassia de Oliveira, Claire Bourlieu-Lacanal, Olivia Ménard, A. Bellanger, F. Carrière, E. Dirson, Yann Le Gouar, P. Pladys, Didier Dupont, Amélie Deglaire

\section{To cite this version:}

Samira Cassia de Oliveira, Claire Bourlieu-Lacanal, Olivia Ménard, A. Bellanger, F. Carrière, et al. Peut-on mimer la digestion gastrique du nouveau-né grâce à un modèle in vitro dynamique ?. Nutrition Clinique et Métabolisme, 2017, 31 (1), pp.45. 10.1016/j.nupar.2016.10.038 . hal-01514236

\author{
HAL Id: hal-01514236 \\ https://hal.science/hal-01514236
}

Submitted on 26 May 2020

HAL is a multi-disciplinary open access archive for the deposit and dissemination of scientific research documents, whether they are published or not. The documents may come from teaching and research institutions in France or abroad, or from public or private research centers.
L'archive ouverte pluridisciplinaire HAL, est destinée au dépôt et à la diffusion de documents scientifiques de niveau recherche, publiés ou non, émanant des établissements d'enseignement et de recherche français ou étrangers, des laboratoires publics ou privés.

\section{다(1)(2)}

Distributed under a Creative Commons Attribution - ShareAlikel 4.0 International 
cette absence de réponse, nous avons étudié, sur un modèle murin d'atteinte musculaire dans un contexte d'hypoxie (HC) et d'inflammation chroniques (IC), les principales voies de signalisation impliquées dans le contrôle de la masse musculaire.

Matériel et méthodes Des rats Wistar ont été exposés à une $\mathrm{HC}$, combiné avec une IC, induite par des instillations intra-pulmonaires de lipopolysaccharides. L'hypertrophie musculaire a été obtenue par l'ablation bilatérale des muscles agonistes du soléaire. Ainsi, en combinant ce modèle de surcharge musculaire à nos conditions environnementales, nous disposons d'un modèle permettant de préciser les rôles perturbateurs de l'HC et de l'IC, rencontrés chez le BPCO, sur les voies de signalisation de la protéosynthèse.

Résultats et analyse statistique Les résultats sont présentés dans le Tableau 1. La pesée des muscles soléaires met en évidence une spécificité de la sensibilité de ce muscle au conditionnement. En effet, l'HC n'a aucune répercussion sur les capacités d'hypertrophie du muscle soléaire alors que l'IC inhibe complètement l'augmentation de sa masse. En outre, HC et IC influencent différemment les protéines impliquées dans les voies de signalisation de la protéosynthèse. En effet, le rapport de la forme phosphorylée/totale de Erk1/2 augmente fortement en HC mais celle-ci est diminuée par l'IC. L'activation d'Akt est elle inhibée par l'HC alors que la protéine S6k1, est très sensible à l'IC indépendamment de l'HC.

Conclusion L'HC et l'IC sont à l'origine de modifications différentes des voies de signalisation de la protéosynthèse du soléaire. Cependant, seule l'IC a des répercussions à la fois sur la masse musculaire et sur les voies de la protéosynthèse avec une modification de Erk1/2, et de S6K1. Cela est dû au fait que l'HC inhibe la voie Akt/mTOR, avec une compensation via la voie des MAPkinase (Erk1/2), afin de maintenir l'augmentation de la masse musculaire. Néanmoins, cette compensation ne semble plus possible lors de l'adjonction d'une IC. L'inflammation semble donc être le stimulus à l'origine du blocage des capacités hypertrophiques du muscle oxydatif, pouvant expliquer la nonréponse à la réhabilitation musculaire de certains patients. Ce modèle semble pertinent pour tester des interventions nutritionnelles destinées à lever cette inhibition, étape importante dans la prise de masse musculaire.

Tableau 1 Effet du conditionnement sur le poids du muscle soléaire (en mg) et sur des protéines de la protéosynthèse du soléaire.

\begin{tabular}{llllll}
\hline & $\mathrm{N}$ & $\mathrm{N}+$ chir & $\mathrm{NLPS}+$ chir & $\mathrm{HC}+$ chir & $\mathrm{HC}+\mathrm{LPS}+$ chir \\
\hline $\begin{array}{l}\text { Muscle soléaire } \\
\text { Protéosynthèse du soléraire }\end{array}$ & $152,9 \pm 5,7$ & $179,3 \pm 11,0^{\mathrm{a}}$ & $153,5 \pm 10,6^{\mathrm{b}}$ & $202,6 \pm 5,9^{\mathrm{a}}$ & $145,8 \pm 3,2^{\mathrm{b}}$ \\
Erk 1/2 & $0,56 \pm 0,14$ & $0,65 \pm 0,16$ & $0,46 \pm 0,11$ & $2,70 \pm 037^{\mathrm{c}}$ & $0,68 \pm 0,11$ \\
Akt & $0,98 \pm 0,13$ & $0,82 \pm 0,17$ & $0,81 \pm 0,12$ & $0,53 \pm 0,08^{\mathrm{d}}$ & $0,55 \pm 0,08^{\mathrm{d}}$ \\
S6k1 & $0,85 \pm 0,15$ & $2,04 \pm 0,15^{\mathrm{a}}$ & $0,97 \pm 0,19$ & $2,19 \pm 0,37^{\mathrm{a}}$ & $0,93 \pm 0,27$
\end{tabular}

Moy \pm SEM ; $n=5-8 ;(p<0,05) ; \mathrm{N}:$ normoxie ; LPS : inflammation ; HC : hypoxie chronique ; HC + LPS : hypoxie chronique + inflammation ; Chir : chirurgie.

a Chir vs. Sham

${ }^{\mathrm{b}}$ LPS \& HC + LPS diff. de N \& HC.

c Diff. de tous.

d Diff. des groupes N.

Déclaration de liens d'intérêts Les auteurs déclarent ne pas avoir de liens d'intérêts.

http://dx.doi.org/10.1016/j.nupar.2016.10.037

\section{Physiologie digestive}

P299

Peut-on mimer la digestion gastrique du nouveau-né grâce à un modèle in vitro dynamique ?

S. De-Oliveira ${ }^{1, *}$, C. Bourlieu ${ }^{1}$, O. Ménard ${ }^{1}$, A. Bellanger ${ }^{2}$, F. Carrière ${ }^{3}$, E. Dirson ${ }^{4}$, Y. Le-Gouar ${ }^{1}$, P. Pladys ${ }^{2}$, D. Dupont ${ }^{1}$, A. Deglaire ${ }^{1}$

${ }^{I}$ UMR 1253 STLO, Inra, France
${ }^{2}$ Service de pédiatrie, CHU de Rennes, Rennes, France

${ }^{3}$ Enzymologie interfaciale et de physiologie de la lipolyse, CNRS, Marseille, France

${ }^{4}$ Unité nutrition et diététique infantile-lactarium, CHU de Rennes, Rennes, France

* Auteur correspondant.

Adresse e-mail : samiracassia.deoliveira@ rennes.inra.fr (S. De-Oliveira)

Introduction et but de l'étude Une meilleure compréhension de la digestion du lait maternel chez le nouveau-né est nécessaire en particulier pour améliorer les formules infantiles et les rendre plus biomimétiques. Pour des raisons éthiques, il est difficile de conduire de telles études chez le nouveau-né. Il est donc nécessaire de développer des modèles pertinents de digestion in vitro. L'objectif de notre étude était de comparer un modèle de digestion dynamique in vitro avec des données obtenues in vivo chez le nouveau-né.

Matériel et méthodes Un digesteur in vitro dynamique (i.e. avec en continu : une régulation du $\mathrm{pH}$, une sécrétion enzymatique et une vidange ; DIDGI ${ }^{\circledR}$, Inra) a été utilisé [1]. Les paramètres du modèle de digestion au stade nouveauné prématuré ont été fixés grâce à une revue exhaustive de la littérature [2] en termes de types et quantités d'enzymes, de flux de sécrétions, de descente en $\mathrm{pH}$ et de vitesse de vidange [2]. Un pool de lait maternel cru ou pasteurisé $\left(62,5^{\circ} \mathrm{C}, 30 \mathrm{~min}\right)$ issu de mères d'enfants prématurés $(n=5)$ a été digéré in vitro. Les digestas gastriques étaient prélevés à intervalle régulier. En parallèle, la digestion de lait maternel cru ou pasteurisé a été suivie chez des nouveaunés prématurés au centre hospitalier universitaire de Rennes (NCT02112331, $n=12$ ). Des prélèvements postprandiaux étaient réalisés à 35,60 ou 90 minutes après le début du repas par la sonde d'alimentation de ces enfants. La structure des digestas (in vitro et in vivo) a été analysée par granulométrie laser et microscopie confocale, la protéolyse par électrophorèse (SDS-PAGE) et la lipolyse par chromatographies en couche mince et gazeuse. Le pH et les volumes des digestas étaient également suivis.

Résultats et analyse statistique Au cours des digestions in vitro et in vivo, une hydrolyse rapide des caséines et une résistance de l'alpha-lactalbumine ont été observées. Un faible degré de lipolyse gastrique (7-19\% à 90 min) a été confirmé in vitro et in vivo. Dans les deux types de digestion, la pasteurisation n' affectait pas les cinétiques ni le degré final de lipolyse et protéolyse mais impactait l'évolution structurale des digestas, en particulier la désintégration de l'émulsion et l'agrégation protéique. Les données de descente en $\mathrm{pH}$ et de vidange gastrique paramétrées dans le digesteur sont à améliorer pour s'approcher au mieux de la physiologie du nouveau-né prématuré.

Conclusion Notre étude a permis de mettre en évidence des conclusions similaires in vitro et in vivo quant à l'impact de la pasteurisation sur la digestion du lait maternel, en termes d'hydrolyse digestive mais aussi en termes d'évolution structurale. Les données in vivo vont permettre d'ajuster au plus près les paramétrages concernant la descente en $\mathrm{pH}$ et la vidange gastrique. Notre modèle sera utile à la fois pour la communauté scientifique et pour les industriels qui s'intéressent à la digestion néonatale et l'optimisation de formules infantiles.

Déclaration de liens d'intérêts Les auteurs déclarent ne pas avoir de liens d'intérêts.

Références

[1] Bourlieu C, et al. Crit Rev Food Sci Nutr 2015;54(11):1427-57.

[2] Ménard O, et al. Food Chem 2014;145:1039-45.

http://dx.doi.org/10.1016/j.nupar.2016.10.038

\section{P300}

Un régime isocalorique riche en protéines

animales ou végétales entrâne une

modification de la composition fécale

associée à un changement du profil

transcriptomique dans la muqueuse rectale chez les volontaires en surpoids

M. Beaumont*, N. Steuer, A. Lan, M. Grausso, A. Marsset-Blaglieri, M. Andriamihaja, N. Khodorova, G. Arinei, D. Tomé, A.-M. Davila,

R. Benamouzig, F. Blachier

UMR0914 PNCA, Inra-Agroparistech, Paris, France

* Auteur correspondant.

Adresse e-mail : martin.beaumont@ hotmail.fr (M. Beaumont) 